\title{
Experimental studies of the details of electron density distribution in a Z-DNA hexamer: new insights, new problems
}

\author{
M. Kubicki ${ }^{1}$, K. Brzezinski², B. Guillot3, M. Jaskolski ${ }^{1,2}$, Z. Dauter ${ }^{4}$ \\ ${ }^{1}$ Faculty of Chemistry, Adam Mickiewicz University in Pozna, Poznan, Poland; ${ }^{2}$ Institute of Bioorganic Chemistry, Polish Academy of \\ Sciences, Poznan, Poland; ${ }^{3}$ Laboratoire de Cristallographie, Institut Jean Barriol, Université de Lorraine, Nancy, Franc; \\ ${ }^{4}$ Macromolecular Crystallography Laboratory, National Cancer Institute, Argonne National Laboratory, Argonne, USA \\ mkubicki@amu.edu.pl
}

The fine details of the electron density distribution, typically far beyond the possibilities of standard X-ray diffraction analysis, can be approached when (ultra)high-resolution diffraction data are available, to allow abandoning the standard model of independent, spherically symmetrical atoms. This more complicated, and much more demanding (both experimentally and computationally) method allows, for instance, to analyze the redistribution of electron density into bonds, intermolecular interactions, etc. Moreover, the AtomsIn-Molecules approach, which is based on the analysis of topological features of high-quality electron density distribution, may offer an insight into the hierarchy of interactions, energetic features, etc. Even though such an approach is well-developed for small molecules, its application in macromolecular crystallography is still under development. Ultrahigh resolution in this case means resolution of at least $0.7-0.65 \AA$. Such data are extremely rare for macromolecular crystals. In addition, modelling problems, disorder, high solvent content, etc., severely limit the number of successful studies of experimental electron density distribution in macromolecules, which so far have been reported for proteins only (e.g. crambin [1], aldose reductase [2] and the high-potential iron-sulfur protein [3]).

For the present study, ultrahigh-resolution diffraction data $(0.55 \mathrm{~A})$ were collected for a Z-DNA hexamer with the sequence $\mathrm{d}(\mathrm{CGCGCG})_{2}$. The results of the high-quality standard refinement [4] suggested that bonding and other features are visible in the difference electron density map (Fig). The quality of these data indeed allows the application of the more sophisticated multipolar model.

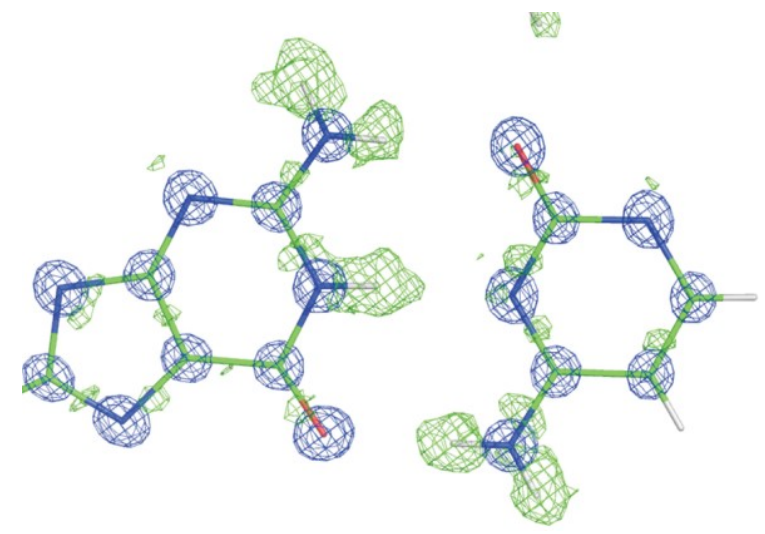

Figure 1. The Watson-Crick base pair C3.G10 with the corresponding $F_{o}$ map (blue, $3 \sigma$ ) and difference $F_{0}-F_{c}$ map (green, $2 \sigma$ ) calculated without the contribution of $\mathrm{H}$ atoms.

The multipolar model has been successfully constructed and the drop in the R factor (and R free) seems to show that real experimental features have been included in this model. The topology of the electron density distribution has been analyzed and the intra- and intermolecular interactions characterized. A number of problems related to the multipolar approach, together with some expected and some unexpected results (e.g. unusual disorder of one of the $\mathrm{C}$ bases) will be presented. We will also consider the practical question concerning this kind of research: is the additional burden and investment of effort justified by the results?

[1] C. Jelsch, M. M. Teeter, V. Lamzin, V. Pichon-Pesme, R. H. Blessing, C. Lecomte. PNAS 97, 3171-3176 (2000).

[2] B. Guillot, C. Jelsch, A. Podjarny, C. Lecomte. Acta Cryst. D64, 567-588 (2008).

[3] Y. Hirano Y, K. Takeda, K. Miki. Nature 534, 281-284 (2016).

[4] K. Brzezinski, A. Brzuszkiewicz, M. Dauter, M. Kubicki, M. Jaskolski, Z. Dauter. Nucleic Acids Res. 39, 6238-6248 (2011).

Keywords: Z-DNA; ultra-high resolution; multipolar model; disorder 